\title{
Tauroursodeoxycholic Acid Protects Nucleus Pulposus Cells from Compression-Induced Apoptosis and Necroptosis via Inhibiting Endoplasmic Reticulum Stress
}

\author{
Wenzheng Wang, Xiangcheng Qing, Baichuan Wang $\mathbb{D}^{\mathbb{D}}$, Kaige Ma, \\ Yulong Wei, and Zengwu Shao \\ Department of Orthopaedics, Union Hospital, Tongji Medical College, Huazhong University of Science and Technology, \\ Wuhan 430022, China \\ Correspondence should be addressed to Zengwu Shao; szwpro@163.com
}

Received 29 October 2017; Revised 14 January 2018; Accepted 30 January 2018; Published 12 March 2018

Academic Editor: Shuang-En Chuang

Copyright (c) 2018 Wenzheng Wang et al. This is an open access article distributed under the Creative Commons Attribution License, which permits unrestricted use, distribution, and reproduction in any medium, provided the original work is properly cited.

Tauroursodeoxycholic acid (TUDCA) is a kind of hydrophilic bile acid, which could protect cells from death via inhibiting endoplasmic reticulum (ER) stress. However, the role of TUDCA in compression-induced intervertebral disc degeneration (IVDD) has not been elucidated. Here, we used a previously described device to mimic in vivo compression conditions. NP cells treated with DMSO or TUDCA were exposed to compression. Then, cell viability, morphology, and apoptosis were detected. Furthermore, apoptosis-related proteins and necroptosis markers were detected too. To investigate the specific cytoprotective mechanisms of TUDCA in IVDD, we detected the ER morphology by electron microscopy. In addition, the ER stress of nucleus pulposus (NP) cells was quantitatively evaluated by analyzing the level of ER-stress-associated proteins. Our results revealed that TUDCA could protect NP cells from excessive compression-induced death by reducing the apoptosis and necroptosis. In addition, ER stress is involved in pathogenesis of IVDD induced by excessive compression and plays a detrimental role. TUDCA exerts its protective functions by inhibiting ER stress. In conclusion, TUDCA could protect NP cells from compression-induced death, which suggested that treatment by TUDCA may be a potential method to retard IVDD.

\section{Introduction}

Intervertebral disc degeneration (IVDD) is responsible for approximately $40 \%$ of low back pain in human [1], which immensely causes society burden in the world. Hence, the research related to the etiology and cure of IVDD is essential. The intervertebral disc (IVD) is a load-bearing component of human body, which is subjected to various mechanical load types. Previous studies have displayed that appropriate mechanical stimulus can exert a protective effect on the IVD $[2,3]$, while excessive mechanical force is proven to be detrimental for IVD $[4,5]$. Excessive compression leads to disc degeneration by inducing cell death and extracellular matrix (ECM) loss $[6,7]$, both of which are central pathological mechanisms for IVDD. However, the exact molecular mechanism by which excessive compression modulates degenerative progression is still not clear.
Tauroursodeoxycholic acid (TUDCA) is a kind of hydrophilic bile acid that is normally produced endogenously in humans at extremely low levels [8]. Multiple studies have demonstrated that TUDCA could protect cells from ERstress-induced death in numerous human diseases such as type 2 diabetes, osteoarthritis, and acute pancreatitis $[8,9]$. In addition, accumulating evidence indicates that TUDCA exerts its biological functions mainly by acting as endoplasmic reticulum (ER) chaperone that could inhibit ER stress and block the activation of unfolded protein response (UPR) [10]. However, the effects of TUDCA on IVDD have not been elucidated before.

The ER is the major organelle responsible for the proper folding of secreted and organelle-targeted proteins. ER stress is referred to as the condition in which cells appear to perform near the functional limits of their secretory pathway capacity and the load imposed on the ER protein-folding 
machinery overwhelms its capability [11]. Several physiological and pathological stimuli cause the accumulation of unfolded proteins in the ER lumen, disrupt ER homeostasis, and trigger ER stress [11, 12]. The unfolded protein response (UPR) is a conserved signal transduction pathway that is activated when cells fail to meet the protein-folding demands under ER stress. It is mediated by three ER-localized transmembrane proteins: double-stranded-RNA-dependent protein kinase- (PKR-) like ER kinase (PERK), activating transcription factor 6 (ATF6), and inositol-requiring enzyme 1 (IRE1). All three UPR transducers are conjugated with the ER-resident chaperone GRP78 and maintained in a nonactive form in resting cells. When ER stress is triggered, GRP78 disaggregates and the UPR pathways are activated [13]. The accumulation of unfolded proteins may be decreased via the attenuation of global mRNA translation and the upregulation of ER chaperones. However, when cells are exposed to prolonged ER stress, they are not able to remove incorrectly folded proteins from the ER and programed cell death is induced. The UPR pathways activate downstream molecules, such as JNK (c-Jun N-terminal kinase) and CHOP (C/EBP homologous protein) and thus induce caspase-4/12-mediated apoptosis [14]. Accordingly, ER stress can play either a protective or a detrimental role in various diseases. ER stress is involved in multiple diseases. In cancer, ER stress can protect tumor cells from apoptosis, promote angiogenesis, and increase the drug resistance of tumors [15]. The core pathogenesis of neurodegenerative disease is the inefficiency of cells to activate UPR and ER-associated degradation [16]. For IVDD, ER-stress-induced disc cell apoptosis was found to be important for the pathogenesis of IVDD [17]. Furthermore, ER stress inhibitors such as TUDCA have been successfully applied for the treatment of some metabolic diseases $[18,19]$. For IVDD, however, whether TUDCA could inhibit compression-induced ER stress in NP cells is still unknown.

Necroptosis is a kind of programmed cell death which shares some common morphological features as necrosis, including the disruption of cell membrane, swelling of organelles, and condensation of chromatin [18]. Necroptosis is a caspase-independent process that is triggered by death receptors, which requires the kinase activity of receptorinteracting protein kinase 1 (RIPK1) and receptor-interacting protein kinase 3 (RIPK3). Necroptosis participates in the pathogenesis of many diseases, including ischemic injury, neurodegeneration, and acute chest syndrome [20]. Our recent study also revealed that necroptosis is involved in the compression-induced cell death of IVDD [21]. Therefore, inhibiting the necroptosis of NP cells may be a potential target for the treatment of IVDD.

In the current study, we first focused on the effects of TUDCA on the survival, cell morphology, apoptosis, and necroptosis of rat NP cells under compression. Furthermore, to determine whether TUDCA exerts its biological functions via inhibiting the ER stress, we observed ER morphology and detected the ER stress level of NP cells for various durations of a compressive force stimulus. Our study revealed protective effects of TUDCA on NP cells for the first time.

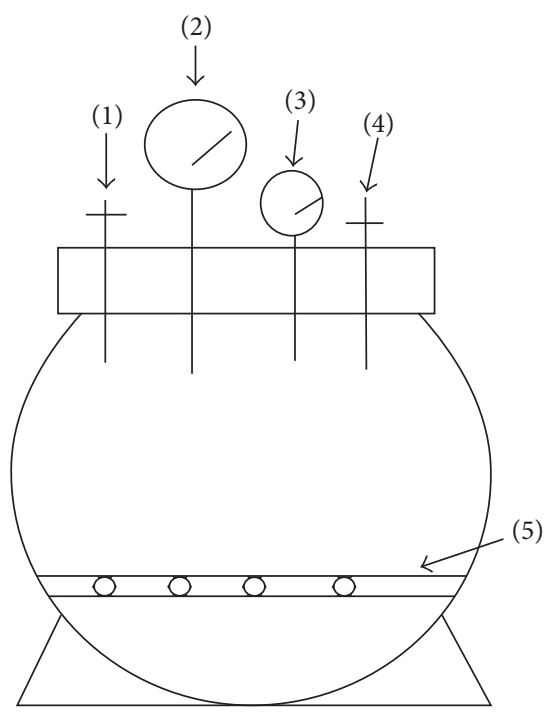

FIGURE 1: Schematic illustration of the custom-made compression apparatus. (1) Air inlet/outlet. (2) Thermometer. (3) Barometer. (4) Safety valve. (5) Bracket.

\section{Methods}

2.1. NP Cells Isolation and Culture. The experimental protocol was approved by the animal experimentation committee of Huazhong University of Science and Technology. SpragueDawley rats (10 weeks old, male) were purchased from the Experimental Animal Center of Tongji Medical College, Huazhong University of Science and Technology. Briefly speaking, rats were euthanized by anesthesia, and the lumbar spines were excised under aseptic conditions. The vertebral appendices and the surrounding soft tissues were thoroughly removed. After the discs were transected from the front edge, the NP tissue was isolated using ophthalmic forceps. The harvested NP tissue was cut into $1 \mathrm{~mm}^{3}$ fragments, and then the fragments were digested with $0.25 \%$ collagenase type II (Invitrogen, Carlsbad, CA, USA) at $37^{\circ} \mathrm{C}$ for $10 \mathrm{~min}$ and filtered through a $70 \mu \mathrm{m}$ filter to remove debris. The obtained cells were seeded in a 6-well culture plate and incubated in Dulbecco's modified Eagle's medium/Ham's F-12 (Gibco, Waltham, MA, USA) with $20 \%$ fetal bovine serum (Gibco) at $37^{\circ} \mathrm{C}$ and $5 \% \mathrm{CO}_{2}$. Cells incubated with $10 \%$ fetal bovine serum at the second passage and maintained in a monolayer were used throughout the experiments.

2.2. Application of a Compression Apparatus to Rat NP Cells. To mimic in vivo conditions, cells were cultured in a previously described compression apparatus made of stainless steel [22]. The pressure apparatus (capacity, 8.2 L) consisted of a sealed can and two meters was constructed to withstand up to $1 \mathrm{MPa}$ of pressure (Figure 1). Rat NP cells were placed in cell culture plates or dishes as monolayer and positioned on the bracket in the sealed can. Sealed can was filled with a small quantity of distilled water at the bottom to maintain moisture. After the sealed can has been fastened down, the compressed gas was pumped into the container until the barometer reached $1 \mathrm{MPa}$. The gas was mixed with $0.5 \% \mathrm{CO} 2$ 
$+9.5 \%$ air $+90 \% \mathrm{~N} 2$ to make sure the partial pressure of $\mathrm{CO} 2$ and air was the same as it was in normal cell culture incubators. At last, the compression apparatus was housed in a cell culture incubator during experiments to maintain a constant temperature of $37^{\circ} \mathrm{C}$.

2.3. Transmission Electron Microscopy. After the indicated treatments, cells in different groups were harvested and fixed in phosphate-buffered saline (PBS) $(\mathrm{pH}=7.4)$ containing $2.5 \%$ glutaraldehyde for $2 \mathrm{~h}$ at room temperature and then postfixed by use of $1 \%$ osmic acid for $1 \mathrm{~h}$ at room temperature. The samples were dehydrated in graded ethanol and embedded in epoxy resin. Then, the samples were cut into $70 \mathrm{~nm}$ sections using the Ultracut UCT Ultramicrotome (Leica, Wetzlar, Germany). Each section was stained with uranyl acetate and lead citrate for $10 \mathrm{~min}$ and observed using the Tecnai G2 12 Transmission Electron Microscope (FEI, Eindhoven, Netherlands).

2.4. Cell Counting Kit-8 (CCK-8) Assays. NP cells were cultured in 96 -well cell culture plates. The seeding density was $5 \times 10^{3}$ cells per well. After $24 \mathrm{~h}$, the original culture medium was changed to the culture medium plus $0.5 \mu \mathrm{l}$ of TUDCA at different concentrations or $0.5 \mu \mathrm{l}$ of DMSO for the control group. The cells were then cultured in the compression apparatus for $18 \mathrm{~h}$. Following the manufacturer's instructions, $10 \mu \mathrm{l}$ of CCK-8 solution (Dojindo, Kumamoto, Japan) and $100 \mu \mathrm{l}$ of serum free medium were added to each well and incubated for $2 \mathrm{~h}$ at $37^{\circ} \mathrm{C}$. The absorbance of each well was determined using the Infinite F50 Microplate Reader (TECAN, Männedorf, Switzerland) at $450 \mathrm{~nm}$.

2.5. Hoechst 33258 Staining for the Observation of Apoptotic Cell Nuclei. Cells were seeded in 6-well cell culture plates at a density of $10^{5}$ cells per well. After treatment with TUDCA at different concentration or DMSO for the control group, cells were immediately cultured in the compression apparatus for $18 \mathrm{~h}$. Then, cells of each group were fixed with $4 \%$ paraformaldehyde for $10 \mathrm{~min}$ and washed with PBS. The cells were stained with $1 \mu \mathrm{g} / \mathrm{ml}$ Hoechst 33258 (Beyotime, Haimen, China) in PBS for $5 \mathrm{~min}$ in the dark. Morphological changes in the nuclei of apoptotic cells were evaluated and photographed under the IX81 Fluorescence Microscope (Olympus, Tokyo, Japan). The excitation wavelength was $350 \mathrm{~nm}$ and the emission wavelength was $460 \mathrm{~nm}$.

2.6. Measurement of Apoptosis by Flow Cytometry and Fluorescence Microscopy. The apoptosis of NP cells was detected using the Annexin V-FITC/PI Apoptosis Detection Kit (Beyotime) and fluorescence microscope. For flow cytometry analysis, NP cells were cultured in 6-well cell culture plates. After treatment with TUDCA or DMSO and culturing in the compression apparatus for $18 \mathrm{~h}$, the total cells including those attached to the plate and those suspended in the culture medium of each group were harvested by trypsinization and centrifugation. Then, cells were washed with PBS and resuspended in a commixture, including $200 \mu \mathrm{L}$ of binding buffer, $5 \mu \mathrm{L}$ of FITC-conjugated Annexin V, and $10 \mu \mathrm{L}$ of propidium iodide (PI) and incubated for $10 \mathrm{~min}$ at room temperature in dark conditions. Apoptosis was detected by the FACalibration flow cytometer (Becton Dickinson, Franklin Lakes, NJ, USA). For fluorescence microscopy observation, compression-treated cells were stained with Annexin V-FITC/PI according to manufacturer's instructions. The double-positive cells after staining with Annexin V-FITC and PI were regarded as apoptotic cells and cells negative for both were nonapoptotic. Apoptotic cells were quantified and represented as the percentage of the total cell count.

2.7. Western Blot Analysis. Cells of various groups were collected and washed with ice-cold PBS. RIPA lysis buffer (50 mM Tris, $150 \mathrm{mM} \mathrm{NaCl}, 1 \% \mathrm{NP}-40,0.5 \%$ sodium deoxycholate, and 0.1\% SDS, Beyotime) plus phenylmethanesulfonyl fluoride (Beyotime) and phosphatase inhibitor cocktail I (Sigma, St. Louis, MO, USA) were used for total protein extraction. The Enhanced BCA Protein Assay Kit (Beyotime) was used to detect the protein concentration of the lysates. Equivalent amounts of protein were loaded onto each well, separated by $10 \%$ or $15 \%$ SDS-PAGE, and then transferred to polyvinylidene fluoride membranes (BioRad, Hercules, CA, USA). The membranes were blocked with 5\% bovine serum albumin (Beyotime) in TBST (a mixture of Tris-buffered saline and Tween-20) for $1 \mathrm{~h}$ at room temperature and incubated overnight at $4^{\circ} \mathrm{C}$ with primary antibodies against p-PERK (\#3179; Cell Signaling Technology (CST), Beverly, MA, USA), p-IRE1 (\#ab48187; Abcam, Cambridge, UK), p-eIF2 $\alpha$ (\#3398S; CST), CHOP (\#2895p; CST), Cleaved caspase-12 (\#ab62484; Abcam), RIPK1 (SAB3500420; Sigma-Aldrich, Germany), pRIPK1 (\#9621; CST), RIPK3 (\#ab62344; Abcam), p-RIPK3 (\#ab195117; Abcam), and GAPDH (Boster, Wuhan, China). After incubation with secondary antibodies (Boster) labeled with horseradish peroxidase for $1 \mathrm{~h}$ at room temperature, immunoreactive bands were developed using BeyoECL Plus (Beyotime). The intensity of bands was quantified using GelPro application.

2.8. Statistical Analysis. Statistical analysis was performed in IBM SPSS Statistics 19. All data obtained from at least three independent assays were presented as means \pm SD. Student's $t$-test was used to analyze differences between the means of two groups. For the CCK-8 assay and the flow cytometry analysis after Annexin V/PI double staining, data was analyzed by one-way ANOVA followed by least significant difference (LSD) tests to analyze differences between control and treatment groups. $P<0.05$ was considered significant.

\section{Results}

3.1. TUDCA Protects NP Cells from Compression-Induced Death. To determine whether TUDCA has protective effects on compression-induced death of NP cells, cell morphology observation and CCK-8 assay were applied. Different concentrations of TUDCA $(0.1,0.3$, and $0.5 \mathrm{mM})$ were used for the low-, mid-, and high-dose groups. Cells treated with isovolumetric DMSO were set as the control group. In each experiment group, TUDCA was dissolved in isometric 


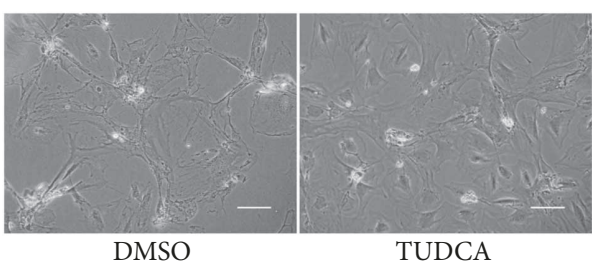

(a)

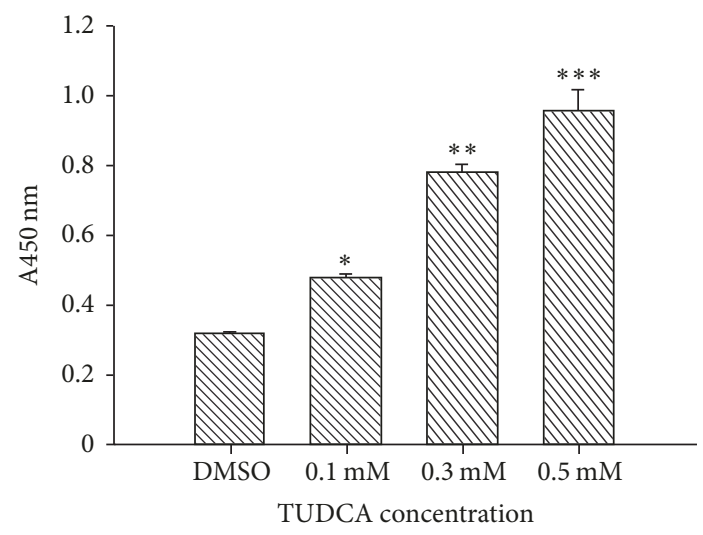

(b)

FIGURE 2: Effects of TUDCA on compression-induced NP cells death. (a) Phase-contrast photomicrographs of morphological differences between the DMSO group and high-dose TUDCA group (magnification $\times 400$, scale bars represent $50 \mu \mathrm{m}$ ). (b) The number of survival NP cells under compression stimulation was detected by CCK- 8 assays. Data are expressed as means \pm SD of three independent experiments $\left({ }^{*} P<0.05,{ }^{* *} P<0.01\right.$, and ${ }^{* * *} P<0.001$ versus DMSO control).

DMSO as control group to exclude the side effect of DMSO. As expected, after exposure to compression ( $1 \mathrm{MPa}$ for $18 \mathrm{~h}$ ), there were obvious morphological differences between the group treated with $0.5 \mathrm{mM}$ TUDCA and the group treated with DMSO. The cells in the DMSO group were shriveled and contact area between cells and the culture plate was reduced; some cells even began to shed from the plate, whereas cells in TUDCA group showed healthier cell morphology (Figure 2(a)). In addition, the CCK-8 results further confirmed that TUDCA treatment could dramatically inhibit compression-induced cell death of NP cells, and this effect was concentration-dependent (Figure 2(b)). Based on above results, we could conclude that TUDCA had protective effects on NP cells under compression.

\subsection{TUDCA Decreases the Apoptosis of NP Cells Induced by} Compression. Apoptosis of NP cells plays important roles in the pathogenesis of IVDD. To explore whether TUDCA could inhibit apoptosis induced by compression, cells were treated as described above and the rate of apoptosis was measured by flow cytometry analysis. Our results demonstrated that, compared with control group, the proportions of early apoptosis cells (Q3) and late apoptosis cells (Q2) as well as the total apoptosis rate were all decreased by TUDCA treatment in a dose-dependent manner (Figures 3(a) and 3(b)). Fluorescence photomicrographs of Annexin V-FITC/PI dual-stained cells also clearly revealed a decrease in apoptotic cells (Figure 3(c)). Moreover, as shown in the photomicrograph of Hoechst 33258-stained cells, in contrast to the control group, the proportion of brightly stained nuclei was diminished by TUDCA (Figure 3(d)). The apoptotic cells in TUDCA group differed significantly compared with the DMSO group (Figure 3(e)).

TUDCA mainly acted as an ER chaperone; meanwhile, the ER pathway is one of the three signaling pathways leading to cell apoptosis. So we detected the level of ER-pathwayrelated proteins $\mathrm{CHOP}$, caspase-12, and cleaved caspase- 12 by using Western blot analysis. As shown in the results, the ER pathway of apoptosis was inhibited by TUDCA in a dosedependent manner (Figure 4). This might partly explain the reason why the cell death was decreased in TUDCA group.

3.3. TUDCA Inhibits the Necroptosis of NP Cells Induced by Compression. Necrostatin-1 (NEC-1) was used as a necroptosis inhibitor because it could particularly inhibit the kinase activity of RIPK1 [18]. To probe the influence of TUDCA on necroptosis, NP cells were divided into four groups: the control group treated with compression only, the group treated with compression and 20 uM NEC-1, the group treated with compression and $0.5 \mathrm{mM}$ TUDCA, and the group treated with compression, NEC-1, and TUDCA. Then, cell viability was detected by CCK- 8 assays. As predicted, NEC-1 and TUDCA could, respectively, promote the survival of NP cells. Nevertheless, there was no statistic difference between the cell viability of TUDCA group and TUDCA + NEC1 group (Figure 5(a)). That means NEC-1 could no longer promote cell viability when TUDCA presented. Therefore, it was logical to speculate that TUDCA could also inhibit the necroptosis of NP cells. To verify our speculation, we detected the level of necroptosis-related biomarkers RIPK1, p-RIPK1, RIPK3, and p-RIPK3 when TUDCA gradient presented. As shown in the results, the necroptosis pathway was inhibited by TUDCA administration in a dose-dependent manner (Figure 5(b)), which explained another reason for the cell viability promoting by TUDCA.

3.4. TUDCA Exerts Its Cytoprotective Effects through Inhibiting the ER Stress. Previous studies have revealed that TUDCA protects cells mainly by inhibiting the ER stress. To clarify whether TUDCA exerts its cytoprotective effects through this mechanism in NP cells too, we first used electron microscopy to examine ER morphological changes. For the morphological changes of ER in compression, as shown in the electron microscope photographs, there were no distinct differences in ER morphology between the $9 \mathrm{~h}$ group and the control group. The ER in the $18 \mathrm{~h}$ group was swollen but 


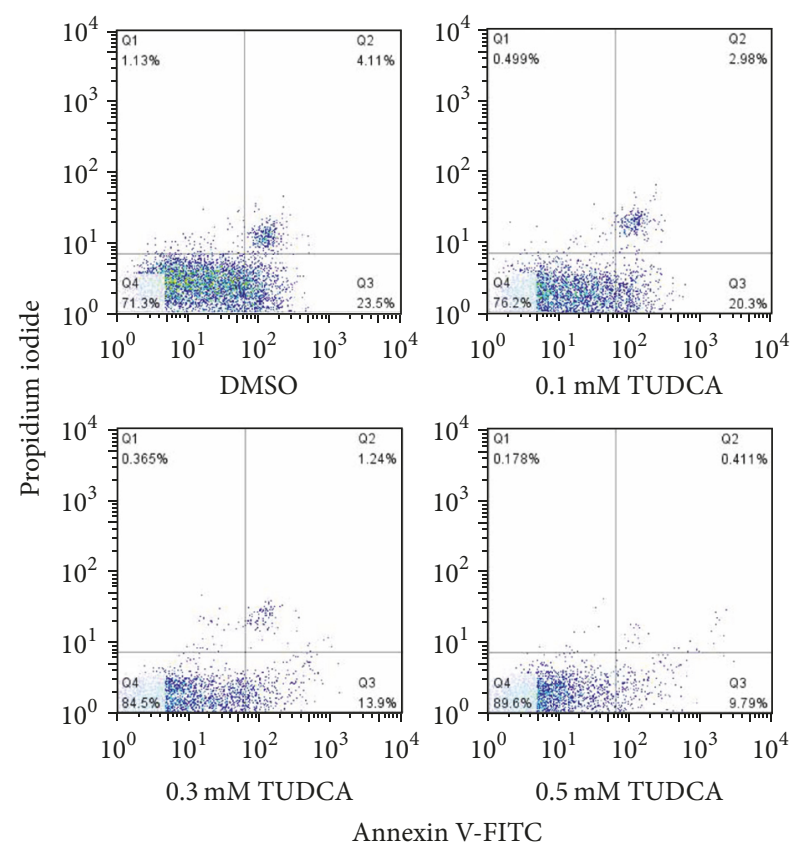

(a)

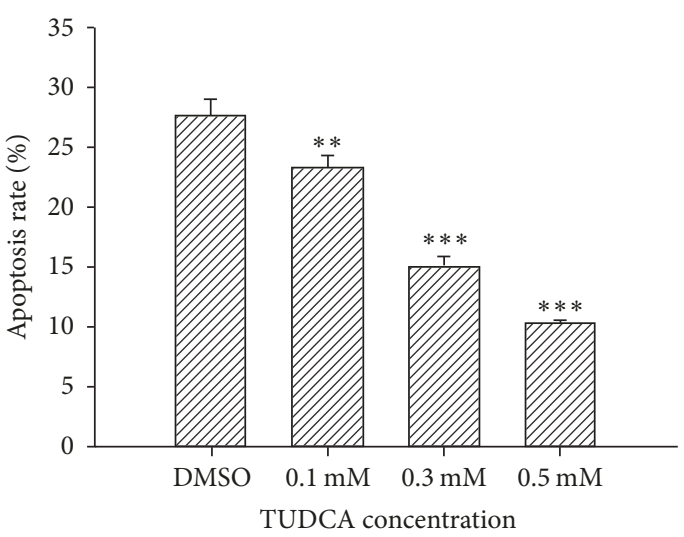

(b)

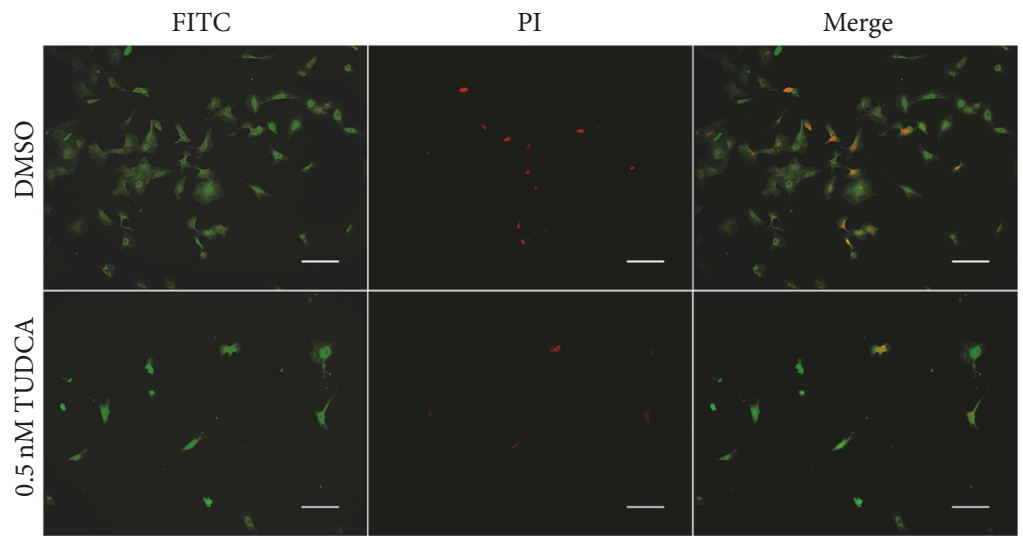

(c)

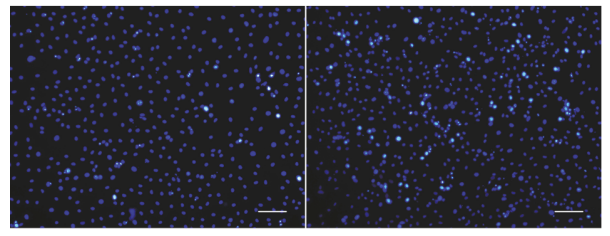

DMSO
TUDCA

(d)

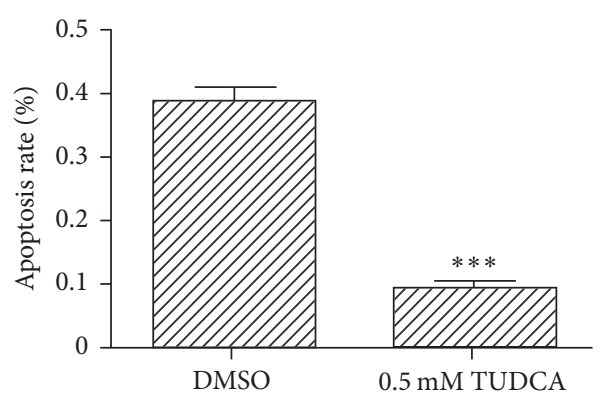

(e)

FIGURE 3: The influence of TUDCA on compression-induced apoptosis of NP cells. (a) Dot graphs obtained by flow cytometry analysis after Annexin V-FITC/PI dual staining. Early apoptotic cells were Annexin V-FITC (+) and PI (-), late apoptotic cells and necrotic cells were Annexin V-FITC (+) and PI (+), and undamaged cells were Annexin V-FITC (-) and PI (-). (b) The apoptosis rate for each group. Data are expressed as means \pm SD of three independent experiments $\left({ }^{* *} P<0.01\right.$ and ${ }^{* * *} P<0.001$ versus DMSO control). (c) Fluorescence photomicrograph of apoptotic cells after Annexin V-FITC/PI dual staining (magnification $\times 200$, scale bars represent $100 \mu \mathrm{m}$ ). The fluorescence photomicrographs of cells stained with FITC (green) and PI (red) were taken under the same visual field and then merged using Image-Pro Plus software. (d) Fluorescence photomicrograph of apoptotic nuclei after Hoechst 33258 staining. Apoptotic nuclei were brightly stained by Hoechst 33258 (magnification $\times 100$, scale bars represent $200 \mu \mathrm{m}$ ). (e) Statistical analysis of the apoptotic rate after Hoechst 33258 staining $\left({ }^{* * *} P<0.001\right.$ versus DMSO control). 


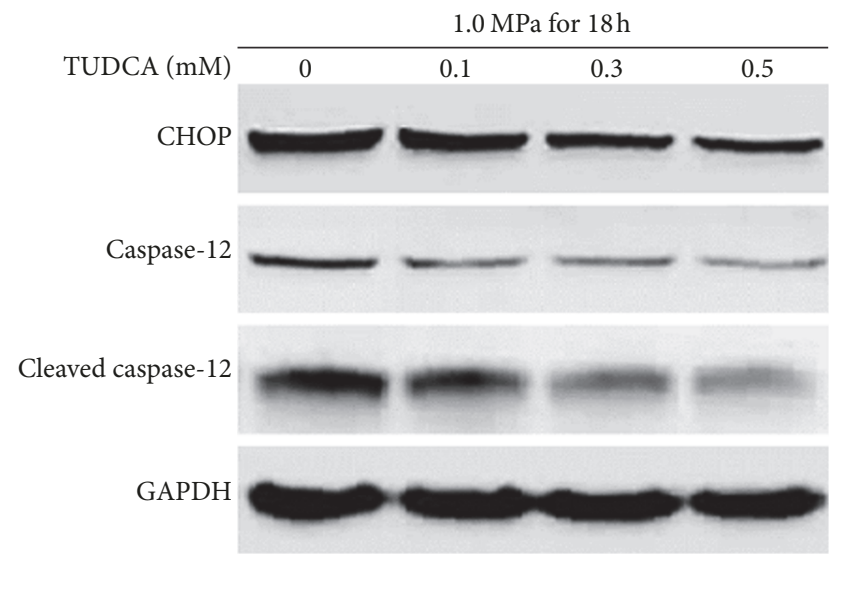

(a)

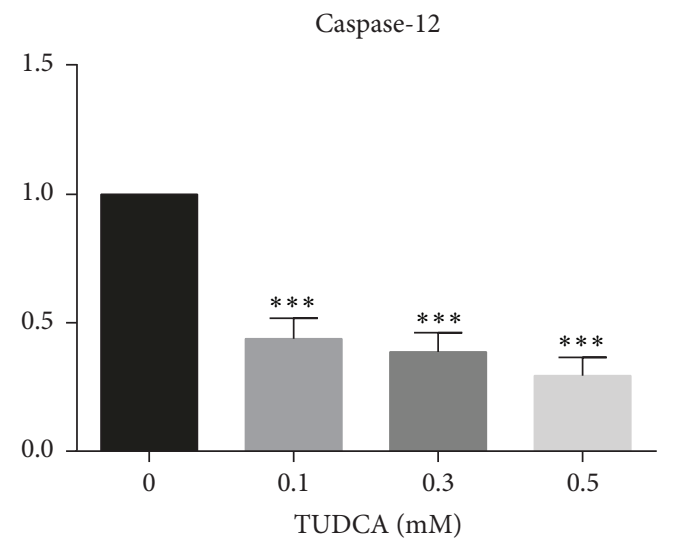

(c)

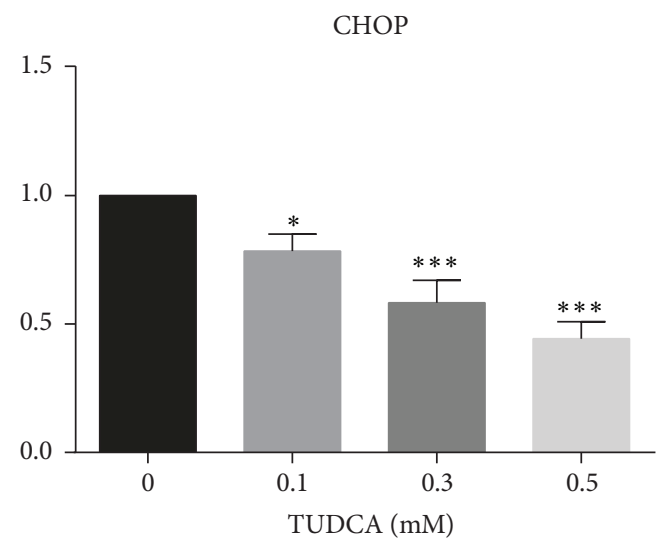

(b)

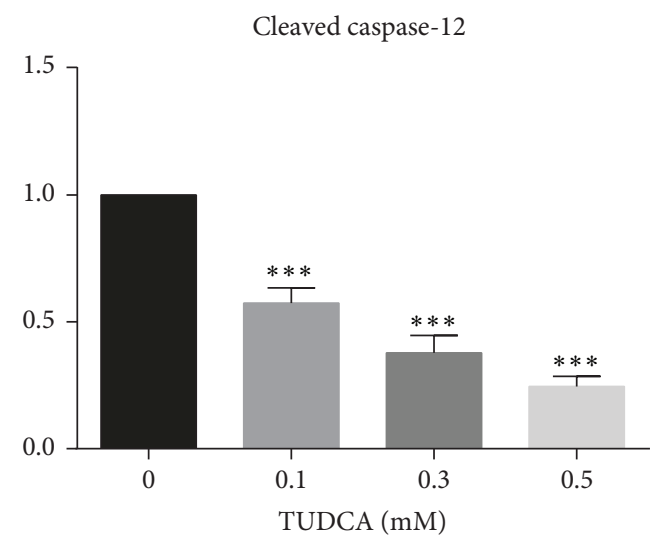

(d)

FIGURE 4: The level of ER-mediated apoptosis-related biomarkers in NP cells treated with DMSO or TUDCA. (a) The Western blot results of ER-mediated apoptosis-related biomarkers CHOP, caspase-12, and cleaved caspase-12. (b), (c), and (d) show the quantitative analysis of expression level of ER-mediated apoptosis-related biomarkers CHOP, caspase-12, and cleaved caspase-12. Results are presented as the fold change in TUDCA-treated groups relative to that in DMSO group $\left({ }^{*} P<0.05\right.$ and ${ }^{* * *} P<0.001$ versus DMSO control).

still maintained a basic reticular form. In the $36 \mathrm{~h}$ group, the ER exhibited extensive swelling and lost its original reticular form (Figure 6(a)). We then compared ER morphological changes between the control group and $0.5 \mathrm{mM}$ TUDCA group. The ER of the control group was swollen, while the ER morphology of $0.5 \mathrm{mM}$ TUDCA group was almost normal (Figure $7(\mathrm{a})$ ). To further verify whether the ER stress level was influenced by compression and inhibited by TUDCA, we used Western blot analysis to detect the expression of ER stress markers. The results showed that expression levels of p-PERK, p-IRE1, and p-eIF $2 \alpha$ increased under compression and were positively related to the compression duration (Figure 6(b)). When cells were treated with different concentration of TUDCA, the expression levels of p-PERK, pIRE1, and p-eIF $2 \alpha$ were all decreased in a concentrationdependent manner (Figure 7(b)). These results demonstrated that ER stress was triggered in compression-induced IVDD and TUDCA could inhibit the compression-induced ER stress of NP cells in a dose-dependent manner.

\section{Discussion}

Mechanical loading is the principal and most extensively investigated pathogenic factor for IVDD. Accumulating studies have revealed that compression could induce NP cell death, which has been believed to play an important role in the pathogenesis of IVDD, via triggering apoptosis and necroptosis and so on $[21,22]$. Therefore, preventing NP cells from compression-induced death is an attracting method for IVDD treatment.

TUDCA is a kind of bile salt with ER chaperone-like properties, which can inhibit ER stress that blocks the activation of UPR owing to its ability to modulate ER function [10]. As a clinical drug approved by the FDA, many investigators have demonstrated that TUDCA can protect cells against various stimuli $[9,23]$. Seyhun et al. has found that TUDCA could reduce endoplasmic reticulum stress, acinar cell damage, and systemic inflammation in acute pancreatitis [9]. Peng et al. also illustrated that preconditioning with TUDCA protects against contrast-induced HK-2 cell apoptosis [10]. 


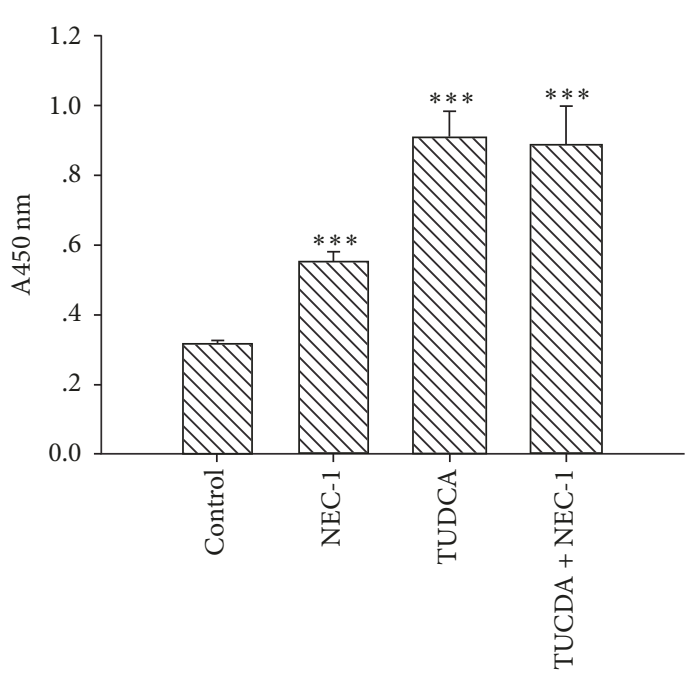

(a)

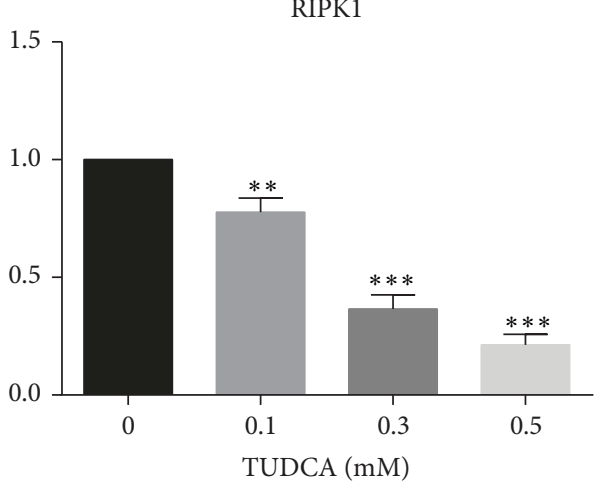

(c)

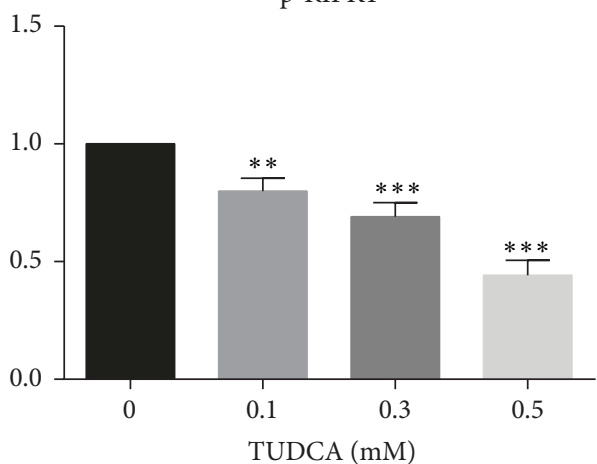

(e)

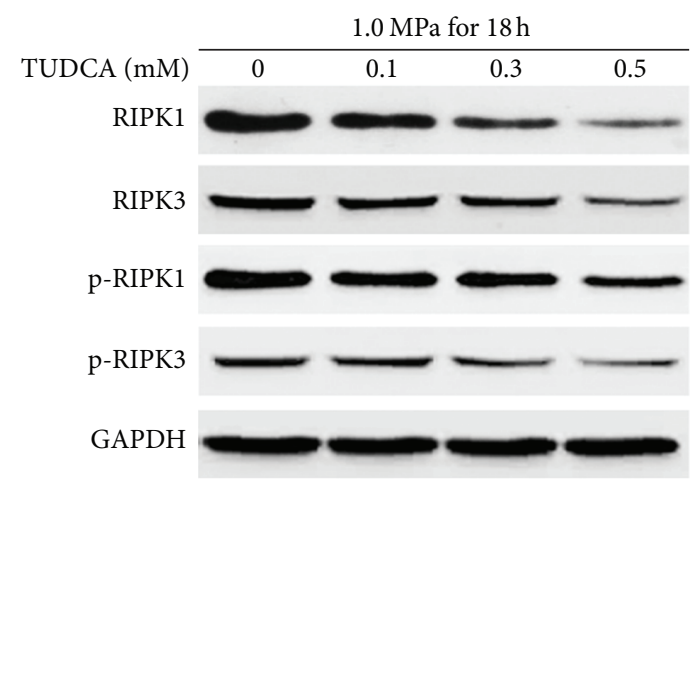

(b)

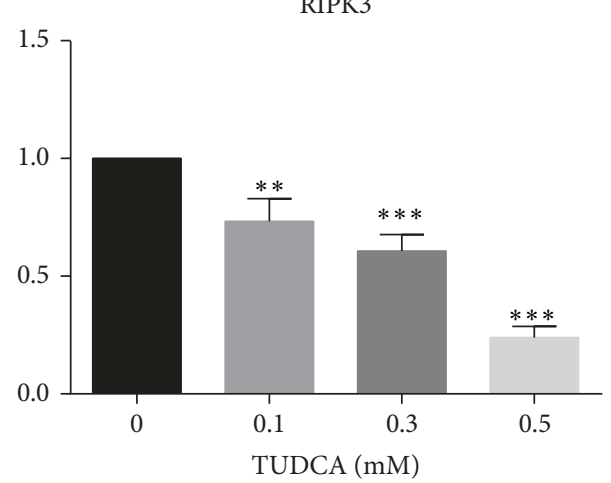

(d)

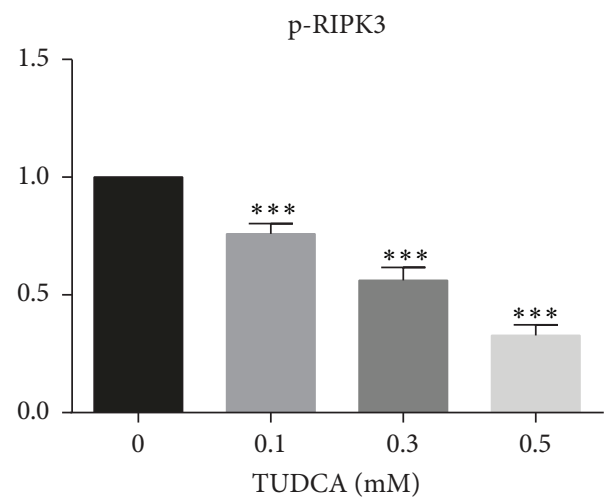

(f)

FIGURE 5: TUDCA reduces compression-induced necroptosis of NP cells in a dose-dependent manner. (a) The NP cells were divided into four groups and the number of survival NP cells was detected by CCK- 8 assays. Data are expressed as means \pm SD of three independent experiments $\left({ }^{* * *} P<0.001\right.$ versus control). (b) The Western blot results of necroptosis-related biomarkers RIPK1, RIPK3, p-RIPK1, and p-RIPK3. (c), (d), and (e) show the quantitative analysis of expression level of necroptosis-related biomarkers RIPK1, RIPK3, p-RIPK1, and p-RIPK3. Results are presented as the fold change in TUDCA-treated groups relative to that in DMSO group. $\left({ }^{* *} P<0.01\right.$ and ${ }^{* * *} P<0.001$ versus DMSO control). 


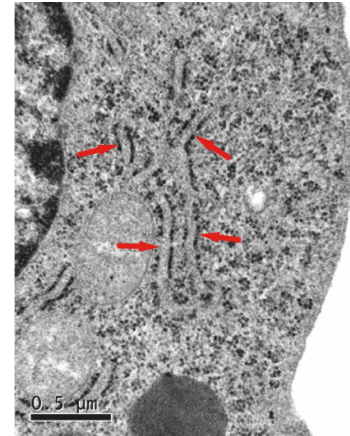

(A)

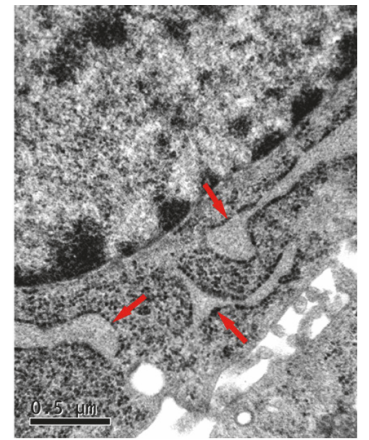

(C)

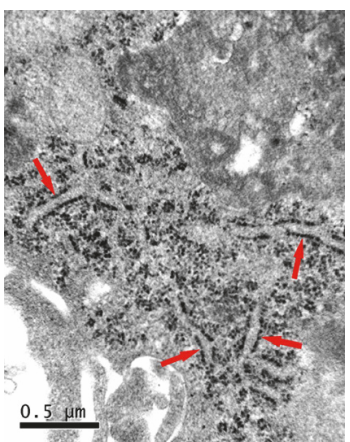

(B)

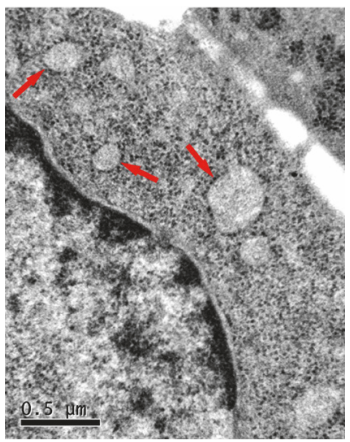

(D) (a)

p-PERK

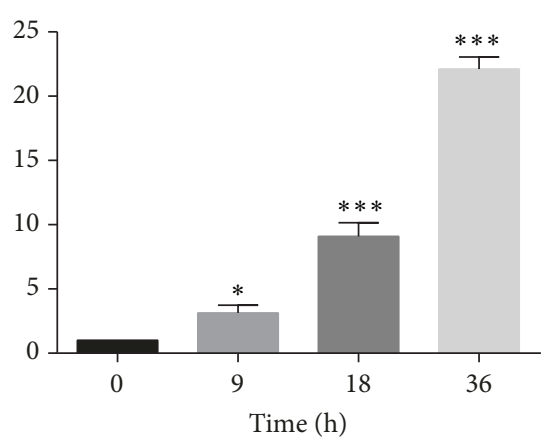

(c)

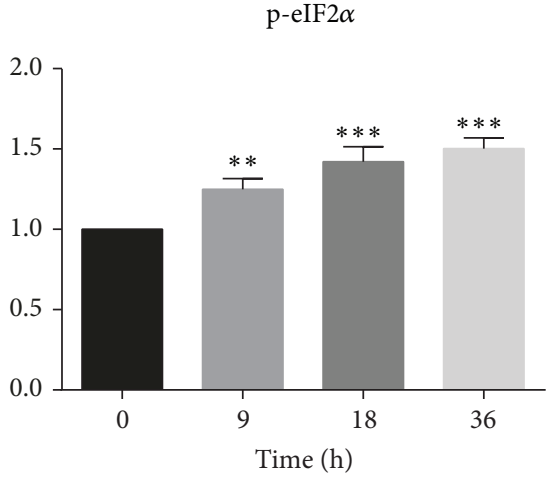

(d)

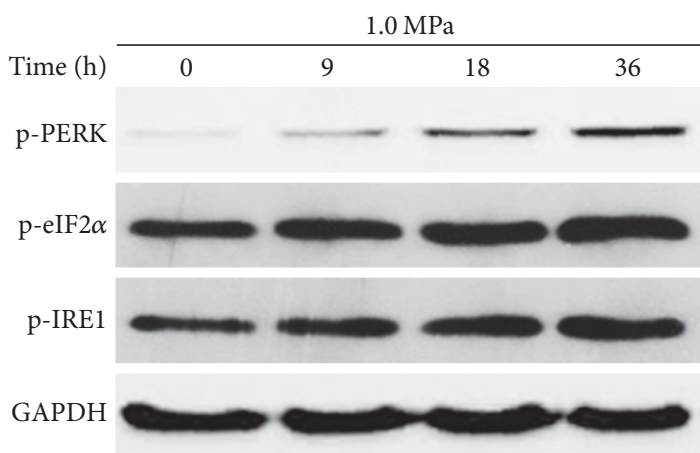

(b)

p-IRE1

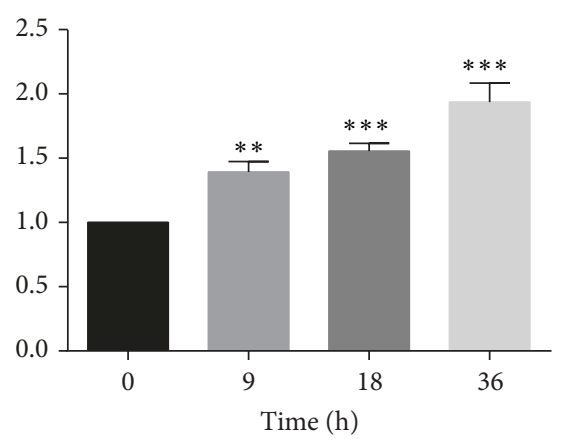

(e)

FIGURE 6: ER stress was triggered in compression-induced NP cell death. (a) Electron microscope photographs of NP cells stimulated by compression for different durations. Control group (A) without exposure to compression. Experimental groups stimulated for (B) $9 \mathrm{~h}$, (C) $18 \mathrm{~h}$, and (D) $36 \mathrm{~h}$. Morphological changes in the endoplasmic reticulum (arrowheads) could be observed by electron microscopy at $26500 \mathrm{x}$. (b) The Western blot results of ER-stress-related biomarkers p-PERK, p-eIF2 $\alpha$, and p-IRE1. (c), (d), and (e) show the quantitative analysis of expression level of ER-stress-related biomarkers p-PERK, p-eIF2 $\alpha$, and p-IRE1. Results are presented as the fold change in 9,18 , and $36 \mathrm{~h}$ groups relative to that in $0 \mathrm{~h}$ group $\left({ }^{*} \mathrm{P}<0.05,{ }^{* *} \mathrm{P}<0.01\right.$, and ${ }^{* * *} \mathrm{P}<0.001$ versus $0 \mathrm{~h}$ control).

Other studies also proved that TUDCA could hinder the development of osteoarthritis, amyotrophic lateral sclerosis, steatohepatitis, and so on $[8,24,25]$. Therefore, to explore whether this cytoprotective function exists in compressioninduced NP cells death, we used TUDCA to treat NP cells. In consistency with previous results, as presented in our article, preconditioning with TUDCA enhanced the survivability of NP cells under excessive compression. The cell population was increased in the presence of TUDCA in a dosedependent manner. In addition, NP cells treated with high dose of TUDCA showed a healthier morphology than the control group. These results verified that TUDCA had protective effects on NP cells under compression. Moreover, our study suggested that treatment by TUDCA may be a potential way to retard IVDD.

ER stress is a condition that causes disturbances in many homeostatic processes and lead to a state in which protein folding slows [26]. However, the role of ER stress is still controversial because ER stress plays a critical and "doubleedged sword-like" role in many diseases. For instance, ER 


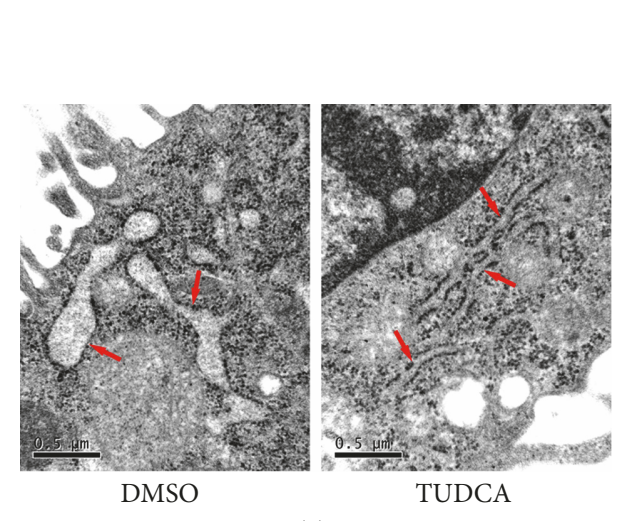

(a)

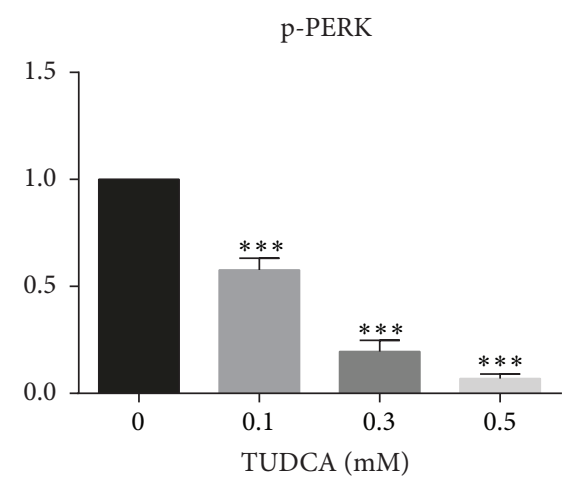

(c)

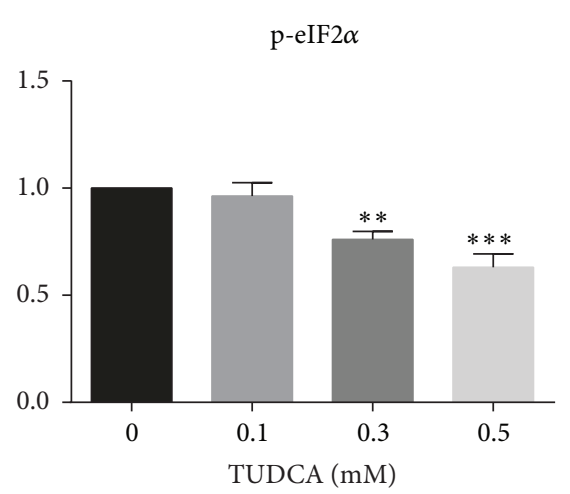

(d)

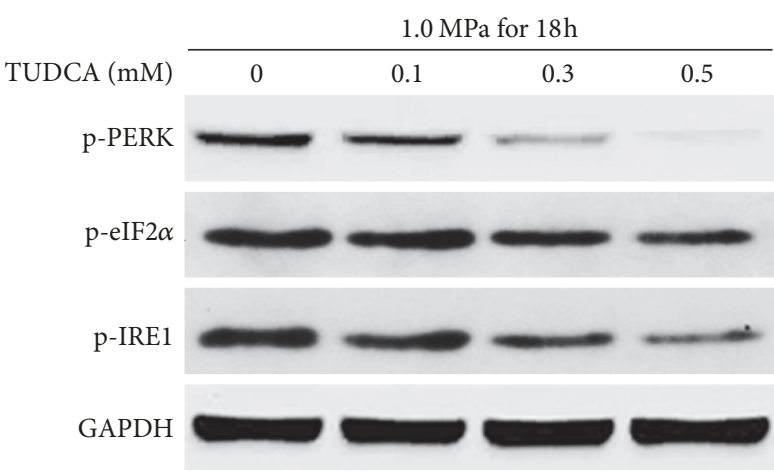

(b)

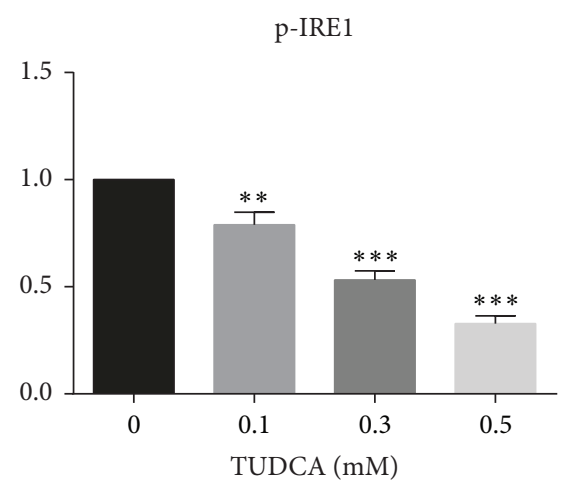

(e)

FIGURE 7: TUDCA inhibits compression-induced ER stress. (a) The ER morphology of the control group and 0.5 mM TUDCA group observed by electron microscope photographs. (b) The Western blot results of ER-stress-related biomarkers p-PERK, p-eIF2 $\alpha$, and p-IRE1. (c), (d), and (e) show the quantitative analysis of expression level of ER-stress-related biomarkers p-PERK, p-eIF2 $\alpha$, and p-IRE1. Results are presented as the fold change in TUDCA-treated groups relative to that in DMSO group $\left({ }^{* *} P<0.01\right.$ and ${ }^{* * *} P<0.001$ versus DMSO control).

stress plays a detrimental role in cancer but a salutary role in neurodegenerative disease as described in Introduction. Former studies have displayed that ER stress is essential for the pathogenesis of IVDD; thus, targeting the underlying molecular events that regulate the ER stress level of NP cells may offer a new therapeutic strategy for IVDD [17]. In this study, we chose suitable compression to mimic the mechanical pressure of the human L4-L5 disc in a standing position while flexed forward [27], and it was equal to the intensity used in our previous studies. We found that compression could induce ER stress in NP cells, which was reflected by morphological changes of the ER. Exposure to compression of $1 \mathrm{MPa}$ for $18 \mathrm{~h}$ resulted in moderate but obvious damage to the ER. The observed damage was more severe and irreversible when the duration was prolonged to $36 \mathrm{~h}$. The Western blot analysis of ER-stress-related biomarkers further confirmed the observation that ER stress was activated after compression. However, the level of p-eIF $2 \alpha$ did not increase for a compression duration more than $18 \mathrm{~h}$. These results implied that compression-induced ER stress was involved in the pathogenesis of IVDD, and a duration more than $18 \mathrm{~h}$ might not further enhance the compression-induced ER stress. Accordingly, we speculated that ER stress was more effective in the middle stage rather than the late stage of IVDD. In an analysis of the apoptosis pathways of human degenerative lumbar discs, the ER pathway was predominantly involved in the mild stage (Pfirrmann grade III) of IVDD [28]. Since TUDCA protects cells from adverse stimulus-induced death via inhibiting ER tress in other diseases, we speculated that TUDCA exerts its cytoprotective effects through the same mechanism. Accordingly, our results showed that preconditioning with TUDCA could attenuate compressioninduced ER morphological changes. Furthermore, ER-stressassociated proteins were also downregulated in comparison with the control group. Hence, we could conclude that ER stress takes part in the pathogenesis of compressioninduced IVDD and TUDCA could protect NP cells from compression-induced death via inhibiting the ER stress.

Apoptosis is one of the most important mechanisms for human IVD cells loss [29]. Therefore, we want to explore whether TUDCA could inhibit compression-induced NP cells death. Through flow cytometry, fluorescence photomicrographs, and Hoechst 33258 staining, we discovered that compression-induced NP cell apoptosis was dramatically inhibited by TUDCA treatment in comparison with control group. This is in accordance with previous researches demonstrating that TUDCA could protect cells from adverse condition-induced apoptosis. There are three apoptosis signaling pathways, namely, the death receptor pathway, mitochondrial pathway, and ER pathway. All three pathways have 
been examined with respect to the apoptosis of IVD cells $[17,30]$. For the ER pathway of apoptosis, numerous studies have shown that the activation of key molecules, such as CHOP, caspase-12, and cleaved caspase-12, is dependent on the activation of UPR signals. Thus, we assumed that TUDCA decreased the compression-induced apoptosis levels by minimizing UPR signals. In accordance with our hypothesis, the Western blotting results showed that ER pathway apoptosisrelated biomarkers including $\mathrm{CHOP}$, caspase-12, and cleaved caspase-12 were all decreased to various level in TUDCA group. These results verified that TUDCA could inhibit the ER pathway of apoptosis induced by compression, which can partly explain why TUDCA showed a protective effect in our research.

The percentage of NP cells with necrotic morphological features increases with age, which testifies that the necrotic cell death of NP cells is precisely controlled by human body [31]. As our recent study revealed, necroptosis is involved in the compression-induced cell death of IVDD and NEC1 could protect cells from necroptosis in a dose- and timedependent manner. In addition, it has also been reported that ER stress could induce ligand-independent TNFR1mediated necroptosis in L929 cells [32]. Considering these facts, we speculated that TUDCA might be able to inhibit compression-induced necroptosis. In our current study, we found that TUDCA could inhibit necroptosis in a dosedependent manner, which suggested that ER stress might be a major upstream pathway of necroptosis in compressioninduced cell death. Recent study has reported that the necroptosis-related proteins, such as mixed lineage kinase domain-like (MLKL) protein and RIPK3, were localized not only at the cell membrane but also on the endoplasmic reticulum of the necroptotic cells [33]. This phenomenon reminded a linkage between ER stress and necroptosis, and inhibition of ER stress could block necroptosis in spinal cord injury. Our study proved that this linkage was also presented in IVDD, but the detailed mechanism of this linkage has to be further researched.

There are several potential limitations related to the present work. First, we only performed monolayer culture of nucleus pulposus cells. However, it is well known that monolayer expansion will change the phenotype of IVD cells, such as decreased gene expression of types II and X collagen and of aggrecan, which might make our results deviate from the real situation [34]. Furthermore, the air pressure used in this study was ten times higher than atmospheric pressure. Although we decreased the concentration of oxygen and carbon dioxide accordingly, which is one-tenth of their concentration in air, this will still more or less influence our results. Therefore, a better in vitro model to mimic the degeneration of IVD should be developed in further studies. In addition, in vivo animal studies should also be adopted in further researches.

\section{Conclusion}

In conclusion, this is the first study to investigate the protective role of TUDCA in the pathogenesis of IVDD. Our discoveries indicated that TUDCA could protect NP cells from excessive compression-induced death by reducing the apoptosis and necroptosis. In addition, ER stress is involved in the pathogenesis of IVDD induced by excessive compression and plays a detrimental role. TUDCA exerts its protective functions by inhibiting ER stress. Our findings could provide a new method for the therapy of IVDD.

\section{Ethical Approval}

The authors applied for the approval of Institutional Review Board Animal Care and Use Committee at Tongji Medical College, Huazhong University of Science and Technology. The experimental protocol was approved by the animal experimentation committee of Huazhong University of Science and Technology and the animals were purchased from the Experimental Animal Center of Tongji Medical College, Huazhong University of Science and Technology.

\section{Conflicts of Interest}

The authors report no conflicts of interest in this work.

\section{Acknowledgments}

This study was supported by the National Key Research and Development Program of China (2016YFC1100100), the Major Research Plan of the National Natural Science Foundation of China (no. 91649204), and the National Natural Science Foundation of China (Grant no. 81572203).

\section{References}

[1] P. Colombier, J. Clouet, O. Hamel, L. Lescaudron, and J. Guicheux, "The lumbar intervertebral disc: from embryonic development to degeneration," Joint Bone Spine, vol. 81, no. 2, pp. 125-129, 2014.

[2] G. Sowa and S. Agarwal, "Cyclic tensile stress exerts a protective effect on intervertebral disc cells," American Journal of Physical Medicine \& Rehabilitation, vol. 87, no. 7, pp. 537-544, 2008.

[3] T. Matsumoto, M. Kawakami, K. Kuribayashi, T. Takenaka, T. Tamaki, and T. Matsumoto, "Cyclic mechanical stretch stress increases the growth rate and collagen synthesis of nucleus pulposus cells in vitro," The Spine Journal, vol. 24, no. 4, pp. 315319, 1999.

[4] C. Wang, S. Gonzales, H. Levene, W. Gu, and C.-Y. C. Huang, "Energy metabolism of intervertebral disc under mechanical loading," Journal of Orthopaedic Research, vol. 31, no. 11, pp. 1733-1738, 2013.

[5] A. Barbir, K. E. Godburn, A. J. Michalek, A. Lai, R. D. Monsey, and J. C. Iatridis, "Effects of torsion on intervertebral disc gene expression and biomechanics, using a rat tail model," The Spine Journal, vol. 36, no. 8, pp. 607-614, 2011.

[6] A. J. L. Walsh and J. C. Lotz, "Biological response of the intervertebral disc to dynamic loading," Journal of Biomechanics, vol. 37, no. 3, pp. 329-337, 2004.

[7] A. H. Hsieh and J. C. Lotz, "Prolonged spinal loading induces matrix metalloproteinase-2 activation in intervertebral discs," The Spine Journal, vol. 28, no. 16, pp. 1781-1788, 2003.

[8] C. Liu, Y. Cao, X. Yang, P. Shan, and H. Liu, “Tauroursodeoxycholic acid suppresses endoplasmic reticulum stress in the 
chondrocytes of patients with osteoarthritis," International Journal of Molecular Medicine, vol. 36, no. 4, pp. 1081-1087, 2015.

[9] E. Seyhun, A. Malo, C. Schäfer et al., "Tauroursodeoxycholic acid reduces endoplasmic reticulum stress, acinar cell damage, and systemic inflammation in acute pancreatitis," American Journal of Physiology-Gastrointestinal and Liver Physiology, vol. 301, no. 5, pp. G773-G782, 2011.

[10] P. Peng, Q. Ma, L. Wang et al., "Preconditioning with tauroursodeoxycholic acid protects against contrast-induced HK-2 cell apoptosis by inhibiting endoplasmic reticulum stress," Angio$\log y$, vol. 66, no. 10, pp. 941-949, 2015.

[11] S. Fu, L. Yang, P. Li et al., "Aberrant lipid metabolism disrupts calcium homeostasis causing liver endoplasmic reticulum stress in obesity," Nature, vol. 473, no. 7348, pp. 528-531, 2011.

[12] S. O. Yoon, D. J. Park, J. C. Ryu et al., "JNK3 perpetuates metabolic stress induced by A $\beta$ peptides," Neuron, vol. 75, no. 5, pp. 824-837, 2012.

[13] B. M. Gardner, D. Pincus, K. Gotthardt, C. M. Gallagher, and P. Walter, "Endoplasmic reticulum stress sensing in the unfolded protein response.," Cold Spring Harbor Perspectives in Biology, vol. 5, no. 3, p. a013169, 2013.

[14] A. M. Gorman, S. J. M. Healy, R. Jäger, and A. Samali, "Stress management at the ER: regulators of ER stress-induced apoptosis," Pharmacology \& Therapeutics, vol. 134, no. 3, pp. 306-316, 2012.

[15] Y. Ma and L. M. Hendershot, "The role of the unfolded protein response in tumour development: friend or foe?" Nature Reviews Cancer, vol. 4, no. 12, pp. 966-977, 2004.

[16] W. Paschen and T. Mengesdorf, "Endoplasmic reticulum stress response and neurodegeneration," Cell Calcium, vol. 38, no. 3-4, pp. 409-415, 2005.

[17] C.-Q. Zhao, Y.-H. Zhang, S.-D. Jiang, L.-S. Jiang, and L.-Y. Dai, "Both endoplasmic reticulum and mitochondria are involved in disc cell apoptosis and intervertebral disc degeneration in rats," AGE, vol. 32, no. 2, pp. 161-177, 2010.

[18] P. Vandenabeele, L. Galluzzi, T. Vanden Berghe, and G. Kroemer, "Molecular mechanisms of necroptosis: an ordered cellular explosion," Nature Reviews Molecular Cell Biology, vol. 11, no. 10, pp. 700-714, 2010.

[19] S. S. Cao and R. J. Kaufman, "Targeting endoplasmic reticulum stress in metabolic disease," Expert Opinion on Therapeutic Targets, vol. 17, no. 4, pp. 437-448, 2013.

[20] S. Singla, J. R. Sysol, B. Dille, N. Jones, J. Chen, and R. F. Machado, "Hemin causes lung microvascular endothelial barrier dysfunction by necroptotic cell death," American Journal of Respiratory Cell and Molecular Biology, vol. 57, no. 3, pp. 307314, 2017.

[21] S. Chen, X. Lv, B. Hu et al., "RIPK1/RIPK3/MLKL-mediated necroptosis contributes to compression-induced rat nucleus pulposus cells death," Apoptosis, vol. 22, no. 5, pp. 626-638, 2017.

[22] F. Ding, Z.-W. Shao, S.-H. Yang, Q. Wu, F. Gao, and L.M. Xiong, "Role of mitochondrial pathway in compressioninduced apoptosis of nucleus pulposus cells," Apoptosis, vol. 17, no. 6, pp. 579-590, 2012.

[23] U. Özcan, E. Yilmaz, L. Özcan et al., "Chemical chaperones reduce ER stress and restore glucose homeostasis in a mouse model of type 2 diabetes," Science, vol. 313, no. 5790, pp. 11371140, 2006.

[24] E.-J. Cho, J.-H. Yoon, M.-S. Kwak et al., “Tauroursodeoxycholic acid attenuates progression of steatohepatitis in mice fed a methionine-choline-deficient diet," Digestive Diseases and Sciences, vol. 59, no. 7, pp. 1461-1474, 2014.
[25] A. E. Elia, S. Lalli, M. R. Monsurrò et al., "Tauroursodeoxycholic acid in the treatment of patients with amyotrophic lateral sclerosis," European Journal of Neurology, vol. 23, no. 1, pp. 45-52, 2016.

[26] R. Iurlaro and C. Muñoz-Pinedo, "Cell death induced by endoplasmic reticulum stress," FEBS Journal, vol. 283, no. 14, pp. 2640-2652, 2016.

[27] H. Y. Fu, T. Minamino, O. Tsukamoto et al., "Overexpression of endoplasmic reticulum-resident chaperone attenuates cardiomyocyte death induced by proteasome inhibition," Cardiovascular Research, vol. 79, no. 4, pp. 600-610, 2008.

[28] H. Wang, H. Liu, Z.-M. Zheng et al., "Role of death receptor, mitochondrial and endoplasmic reticulum pathways in different stages of degenerative human lumbar disc," Apoptosis, vol. 16, no. 10, pp. 990-1003, 2011.

[29] C.-Q. Zhao, L.-S. Jiang, and L.-Y. Dai, "Programmed cell death in intervertebral disc degeneration," Apoptosis, vol. 11, no. 12, pp. 2079-2088, 2006.

[30] J.-B. Park, K.-W. Kim, C.-W. Han, and H. Chang, "Expression of Fas receptor on disc cells in herniated lumbar disc tissue," The Spine Journal, vol. 26, no. 2, pp. 142-146, 2001.

[31] J. A. Buckwalter, "Aging and degeneration of the human intervertebral disc," The Spine Journal, vol. 20, no. 11, pp. 1307-1314, 1995.

[32] S. Saveljeva, S. L. Mc Laughlin, P. Vandenabeele, A. Samali, and M. J. Bertrand, "Endoplasmic reticulum stress induces ligandindependent TNFR1-mediated necroptosis in L929 cells," Cell Death \& Disease, vol. 6, no. 1, Article ID e1587, 2015.

[33] H. Fan, H.-B. Tang, J. Kang et al., "Involvement of endoplasmic reticulum stress in the necroptosis of microglia/macrophages after spinal cord injury," Neuroscience, vol. 311, pp. 362-373, 2015.

[34] T. Kluba, T. Niemeyer, C. Gaissmaier, and T. Gründer, "Human anulus fibrosis and nucleus pulposus cells of the intervertebral disc: effect of degeneration and culture system on cell phenotype," The Spine Journal, vol. 30, no. 24, pp. 2743-2748, 2005. 


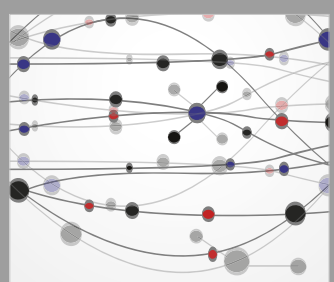

The Scientific World Journal
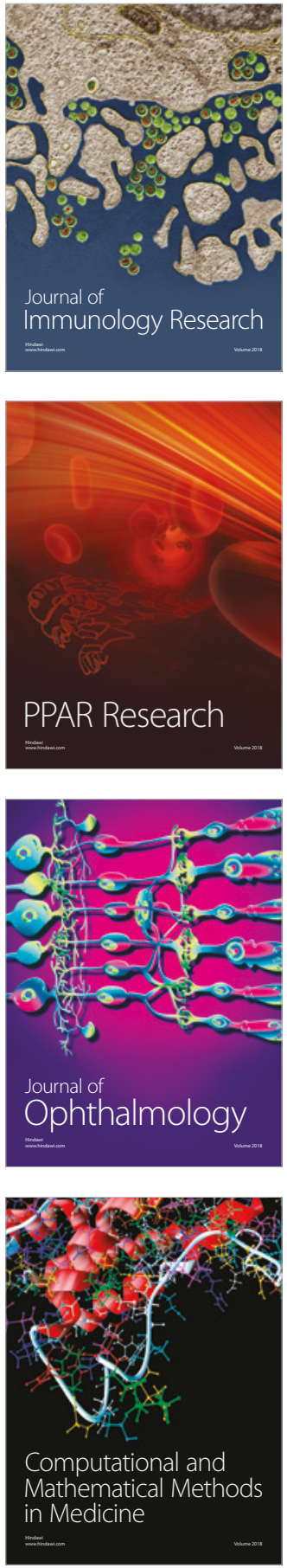

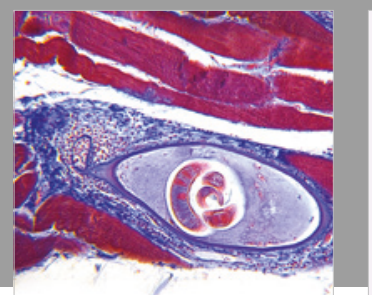

Gastroenterology Research and Practice

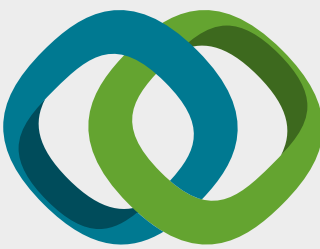

\section{Hindawi}

Submit your manuscripts at

www.hindawi.com
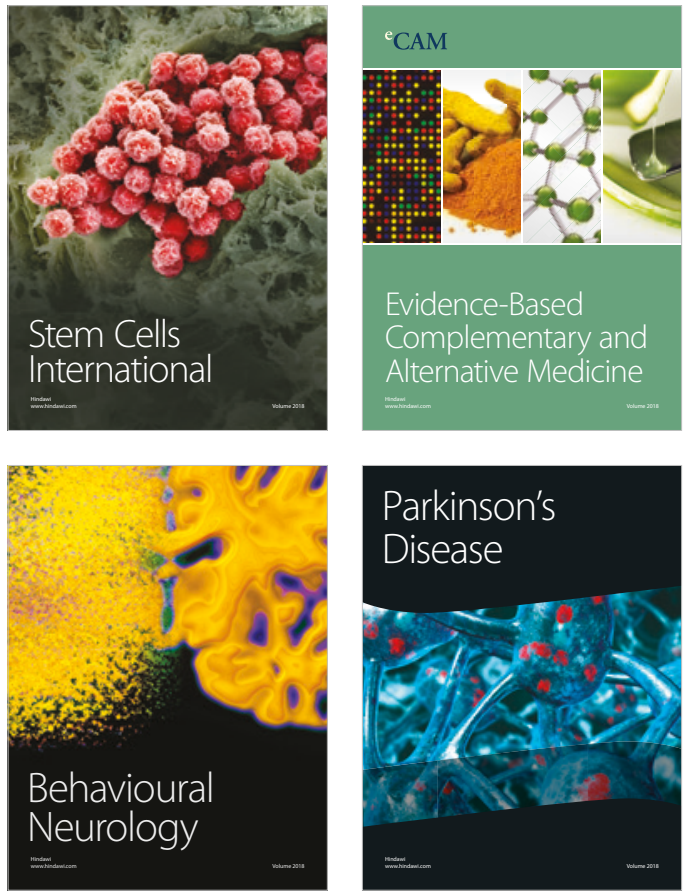

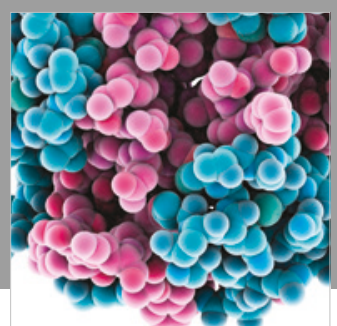

ournal of

Diabetes Research

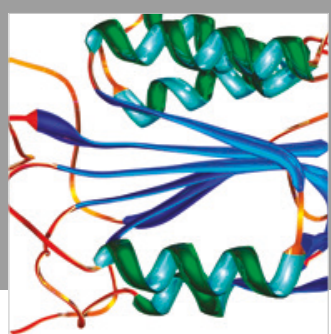

Disease Markers
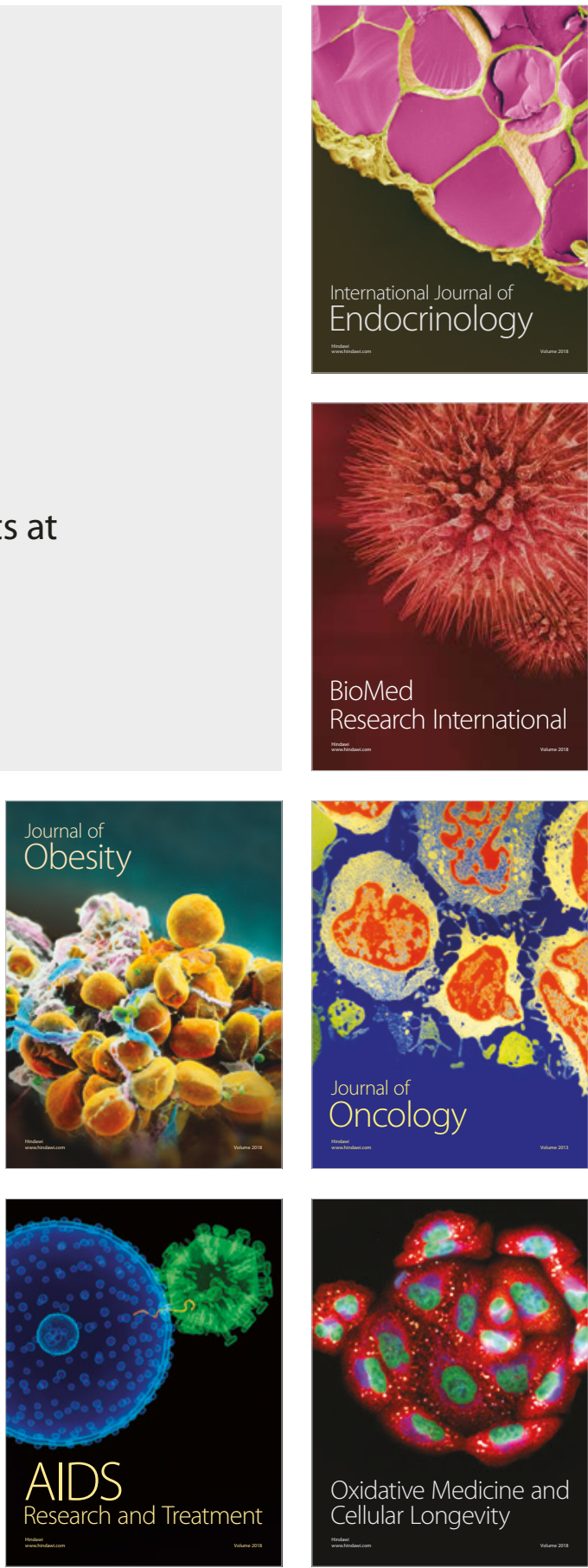